\title{
Problems of dynamometer construction for wheelchairs and simulation of push motion
}

\author{
Bartosz Wieczorek ${ }^{1, *}$, and Lukasz Warguła ${ }^{1}$ \\ ${ }^{1}$ Poznan University of Technology, Chair of Basic of Machine Design, Piotrowo Street 3, 60-965 \\ Poznan, Poland
}

\begin{abstract}
The article discusses the problem of the construction of a test stand that allows simulation of wheelchair propulsion in various terrain conditions. The described test stand is a complete measuring system which allowing to analyse the parameters of the biomechanical system humanwheelchair. The described test stand allows observation of biomechanical parameters such as: centre of gravity, muscle activity, path, speed and acceleration. An important aspect of test stand is to replicate the real wheelchair motion in to laboratory conditions. The effect of the considerations taken in the article is the development of the structure, the construction of the test stand and the development of a research method using the stand.
\end{abstract}

Keywords: wheelchair, propulsion, dynamometer

\section{Introduction}

Wheelchairs are „man-machine" systems involving a close interrelation between a human and a piece of technical equipment. Only joint and smooth cooperation of these two elements makes it possible for the system to accomplish the locomotion function. There are various types of wheelchairs, among which manually propelled wheelchairs are the group most common in use. Wheelchairs of this type are user friendly and inexpensive and are a good alternative for ensuring mobility to people with disabilities. The drawback of these designs is that they have not been modified over the years. Therefore there arises a need to further develop manually propelled wheelchair designs to improve their performance without compromising their compactness and ease of operation.

To solve the above-defined problem a research team was set up whose main task was to investigate the biomechanics of manual wheelchair propulsion, with focus on the analysis of the parameters of the human-wheelchair anthropotechnical system. To accomplish the objective defined it is necessary to perform a number of constituent research tasks, which include:

- engineering of a test stand needed to perform research on the mechanics of wheelchair motion,

\footnotetext{
*Corresponding author: bartosz.wieczorek@put.poznan.pl

Reviewers: Michat Bartyś, Juraj Gerlici
} 
- setting up of necessary procedures and methodology of experimental research on wheelchair propulsion biomechanics, focusing in particular on the user's muscular activity, - building of mathematical models of wheelchair motion for thorough analysis of propulsion parameters,

- engineering of prototypes of innovative wheelchair design proposals,

- developing of solutions with regard to wheelchair ergonomics and driving comfort of wheelchair users.

This paper tackles the issues of design and construction of a wheelchair test stand. It contains the description of the test stand's structure and the difference between the wheelchair motion in real life conditions and its motion at the test stand. The key problem addressed by the team was the method of simulation of wheelchair motion at the test stand and of biomechanical parameters using available technical means.

\section{Motion of wheelchair in real life conditions and at the test stand}

Under real life conditions movement of a wheelchair is comprised of three stages: acceleration, constant speed motion and deceleration [1]. These phases have an effect on wheelchair speed at a given time, as shown in Fig. 1. The acceleration and constant motion phases are in turn subdivided into drive and return phases [2,3]. During the drive phase the wheelchair operator's upper extremity is in contact with the propulsion element and at the same time it is transferring muscular power [4] to the drive wheel turning it into the wheelchair propulsion force. Operation of the propulsion force results in supplying the system with positive acceleration.

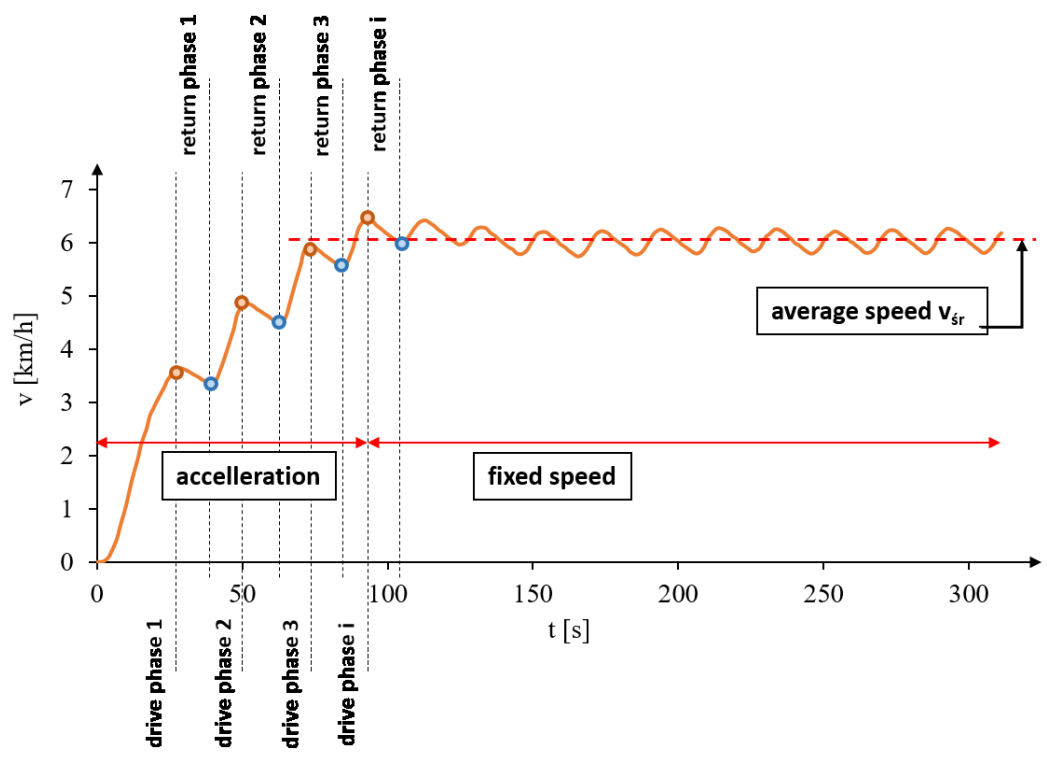

Fig. 1. Change of speed over time during wheelchair propulsion

In the return phase the operator's upper extremity returns to the starting position (Fig. 2). The drive phase is terminated when the propelled element has turned by a certain angle. The value of this angle results from the operator's upper extremity range of motion and from the geometric dimensions of the wheelchair's propulsion system. The drive phase is followed by the return phase in which the operator's hand returns to the starting position. 


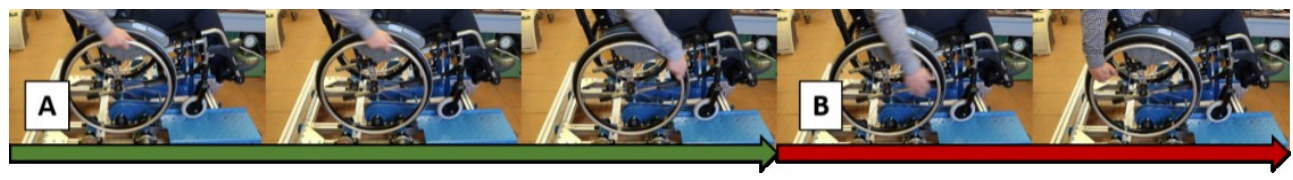

Fig. 2. Time-lapse image of propulsion movement with an indication of the drive phase (A) and return phase (B)

During the return phase no propulsion force is generated and the wheelchair is continuing its movement by inertia and owing to energy accumulated in the drive phase. During this phase the wheelchair also decelerates due to resistances to motion. The resultant acceleration of deceleration $a_{h}$ reduces wheelchair's velocity in the return phase. This makes the operator repeat the drive phase to increase the speed or to maintain constant average driving speed $v_{s}$. It needs to be stressed, that under real life conditions the value of acceleration of deceleration and the duration of the return phase do not bring the wheelchair to a complete stop. They result in only a few percent drop in speed compared to the maximum speed of the wheelchair recorded during the drive phase. As a result the operator can accelerate the wheelchair to a certain average speed and then maintain this speed over a prolonged period of time.

During the return phase the speed of the wheelchair can drop to zero if the drive phase is not repeated for a long enough time. This is due to the acceleration of deceleration stopping the wheelchair (1). Depending on the value of acceleration of deceleration the duration of the return phase $t_{f p}$ may increase. The acceleration of deceleration depends on the place of wheelchair use, which determines the strength of the resisting forces [5]:

$$
v(t)=v_{0}^{i}-a_{h}^{i} t_{f p}
$$

where:

$v(t)$ - actual speed of the weelchair,

$v_{0}{ }^{i}$ - linear speed of the wheelchair on completion of the i-th propulsion phase,

$a_{h}{ }^{i}$ - acceleration of deceleration in the i-th return phase,

$t_{f p}$ - duration of propulsion phase.

It needs to be stressed that if the wheelchair travels on an even surface acceleration of deceleration is zeroed when the wheelchair stops. While in the case of motion uphill after braking of forward motion acceleration of deceleration causes the wheelchair to move backwards.

Investigation of wheelchair propulsion at a test stand makes it possible to propel the wheels of the vehicle without effecting its linear movement. As a result no inertia force due to acceleration of the wheelchair during the drive phase occurs in the system. Inertia force is one of the forces acting on the wheelchair, which sustains its motion during the return phase. During wheelchair testing conducted at the test stand the only force to sustain the drive wheel revolutions during the return phase is the energy resulting from the inertia of the revolving drive wheel. The adopted test stand concept includes the elements introducing to the contemplated human - machine system additional resisting forces, other than these acting in real life conditions, and at the same time it excludes action of wheelchair inertia force. As a result during the drive phase the system is unable to accumulate sufficient energy to keep the drive wheel revolutions up throughout the return phase as it happens during real life use (2):

$$
v(t)=\omega(t) \cdot R_{D} \cdot 60 .
$$

The effect of the lack of inertia force during the tests and of reduction of the test stand's internal resistances is depicted in the graph representing the theoretical change of speed over time (Fig. 3). The graph shows a situation in which the speed is increasing during the drive phase and immediately dropping down to zero on the completion of this phase (Fig. 3b). This 
is due to the occurrence of internal resistances within the test stand system and to lack of inertia force in the linear motion.

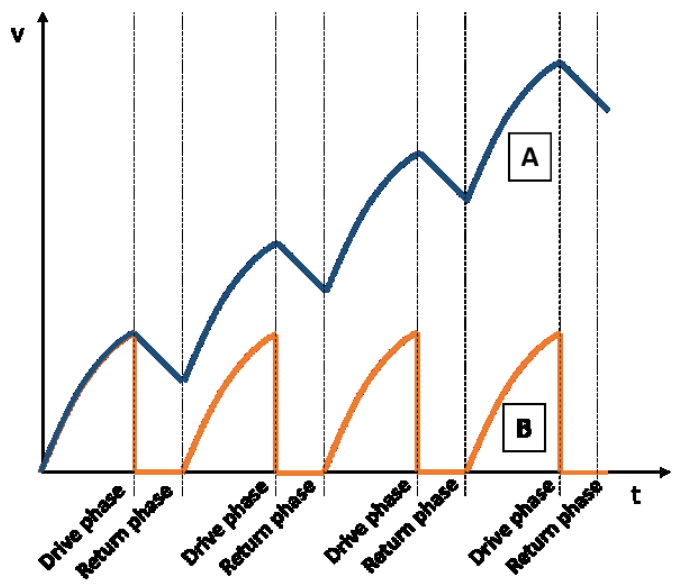

Fig. 3. Graph of the wheelchair's theoretical speed under real life conditions (A) and at the test stand with no compensation for the lack of inertia (B)

\section{Design of the research test stand}

The investigated human - machine system is comprised of three main components: the human, the tested wheelchair and the test stand (Fig. 4). Parameters occurring within the system were subdivided into internal, measured and input parameter groups. The internal parameters result from internal correlations between individual components of the system.

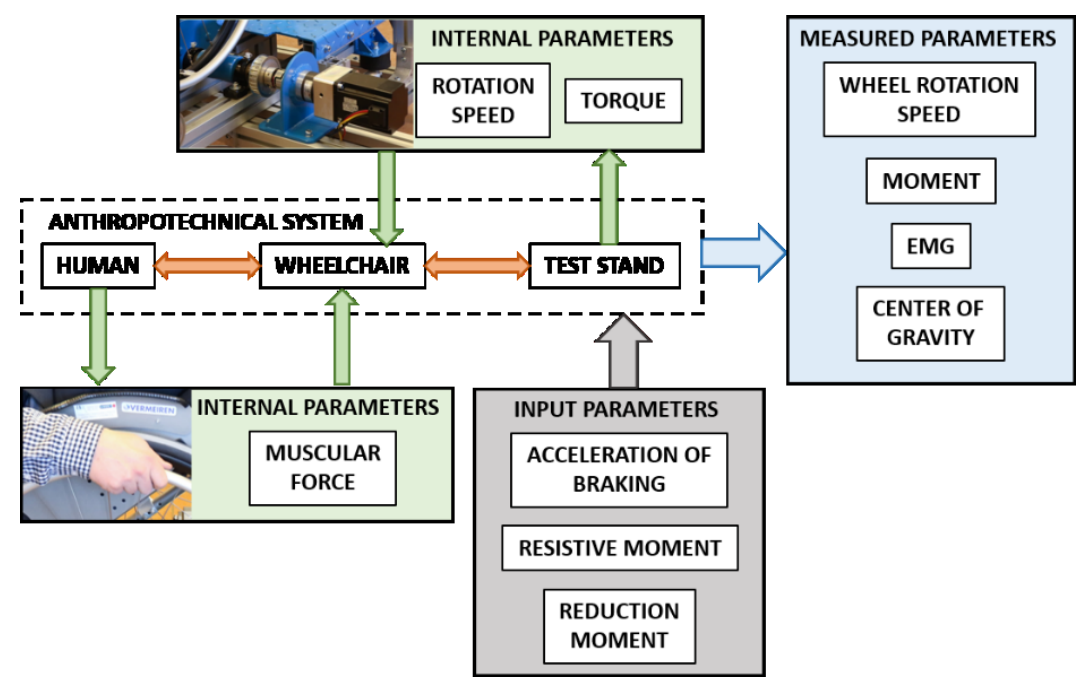

Fig. 4. Diagram of the conveyance of dynamic and biomechanical parameters within the system of the test stand for investigation of propulsion biomechanics of manual wheelchairs

They include muscular power propelling the wheelchair hand rims and rotational speed and torque generated by the wheelchair drive wheel and transferred to the driving rollers of the test stand. The measured parameters are collected from the test stand component and from 
the human component of the system. The human component supplies the information on electromyographic muscle activity (EMG). The other parameters such as rotational speed, torque, location of the system's centre of gravity are collected from the test stand component. The main test stand input parameter is acceleration of deceleration defining the force of resistances to motion in the return phase of the propulsion cycle. Other input parameters include the torque of resistances resulting from the simulated terrain and reduction torque reducing the test stand's internal resistances.

The key element of the presented human-machine system is the test stand component. It is an element which bonds the man and the wheelchair for the purpose of testing and allows to measure and simulate biomechanical parameters. This component has been designed, patented and built [6]. The test stand has been designed to be able to take the revolutions of the wheelchair wheels and to simulate its tilt. However, most importantly, it is equipped with an array of sensors for the measurement of basic biomechanical parameters.

The main element of the test stand (Fig. 5) is the support frame (1) with the weighing pan (3) mounted on it using strain-gauge balances (2). Strain gauge balances make it possible to measure loads applied by the wheelchair with the operator seated in it to four points on the weighing pan (3).

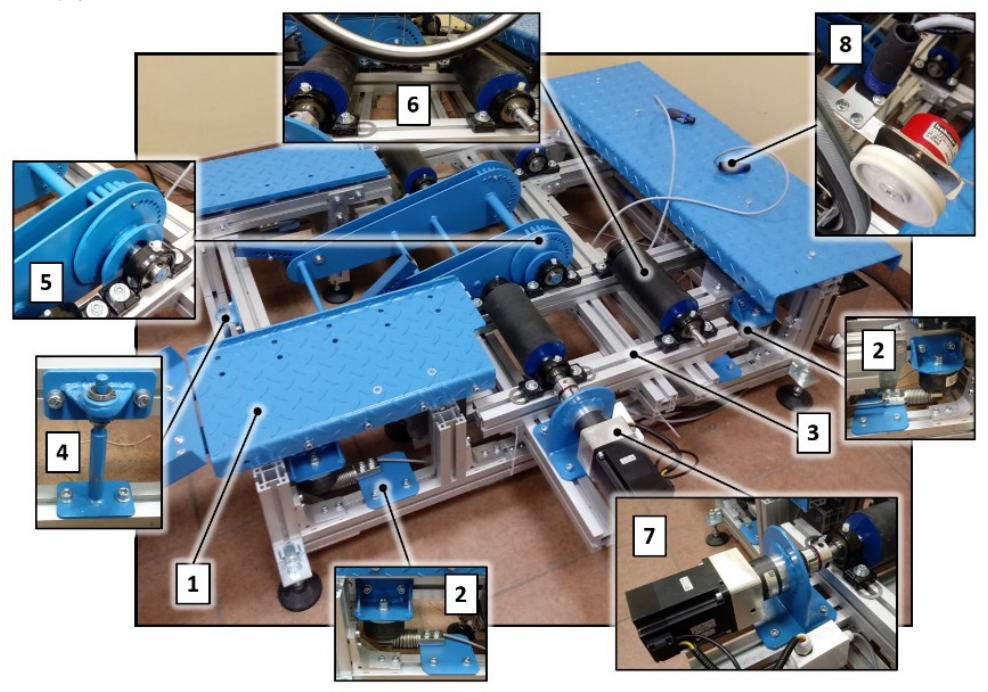

Fig. 5. View of the designed test stand, detailing the key elements

Based on these measurement the location of the centre of gravity of the human wheelchair system was determined. Due to the sensitivity of the strain gauge balances to longitudinal loads 3 linear guide rails were used, which allow only for vertical movement of the weighing pan in relation to the support frame. The weighing pan was provided with a system for wheelchair frame fastening (5) and with two traction roller assemblies (6). The system for wheelchair frame fastening shown in Fig. 5 is comprised only of a lever making it possible to tilt the wheelchair in sagittal plane. The driving rollers assembly is made up of two rubber coated rollers of truncated cone shape tapering off towards the centre of the test stand. The rollers receive torque from the drive wheels, but they can also generate excitations. The rear roller in the assembly is an idler and its sole function is to support the wheelchair. The front roller is powered by a BLCD motor which makes it an active roller. Its function within the system is to reduce internal rolling resistances and it can also generate excitations. In order to register the distance covered and speed values two encoders (8) were installed within the test stand component to take independent measurements on the left and right wheel. 
The method of the wheelchair's installation on the test stand is shown in Fig. 6. To be mounted at the test stand the wheelchair needs to be rolled up the approach ramp and positioned on the test stand in such a way that its rear wheels are sitting on driving rollers (A). Then, the system for wheelchair frame mounting (F) should be fastened to the wheelchair. This is done using clamps (D) equipped with rubber cylinders. It is important that this joining element should be compliant as its compliance makes it possible to tilt the wheelchair and allows for self-alignment of wheelchair frame in relation to driving rollers. The wheelchair frame fastening system is provided with two independent adjustment possibilities. The first adjustment possibility refers to changing the inclination of the weighing pan lever (B). The desired tilt angle is obtained using the perforated disc and safety pin (C). This adjustment makes it possible to simulate uphill or downhill wheelchair motion. The other adjustment possibility (E) allows to adjust the lever arm height to the height of the wheelchair frame.

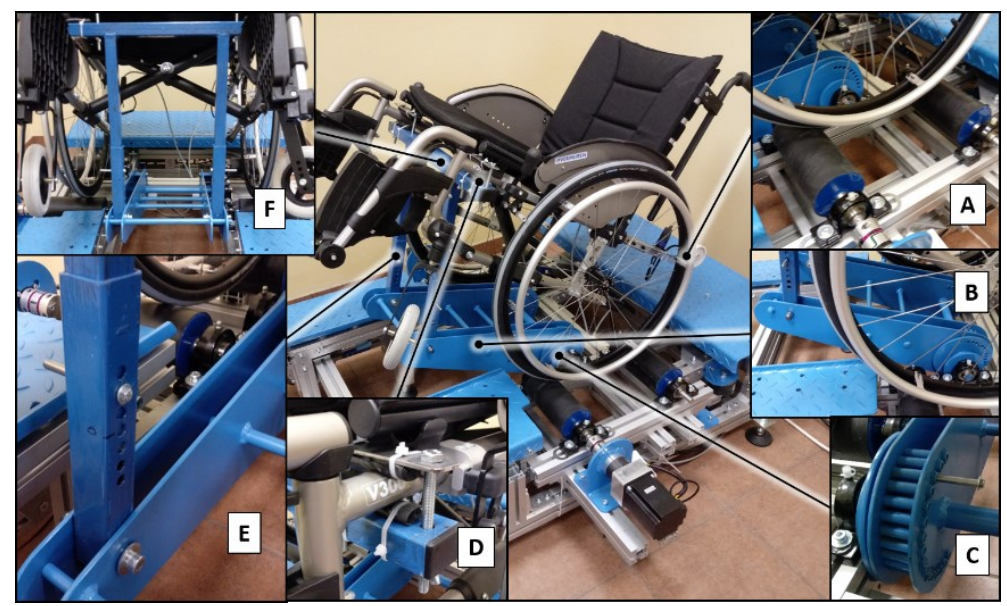

Fig. 6. View of the designed test stand, detailing the key elements

The above-specified elements of the test stand system can be additionally supplemented with the system for evaluating and recording the electromyographic activity of muscles (EMG). This is a separate module which can work independently from the test stand and is not its integral part but just an addition to it. Nevertheless it is planned to be used in the course of the testing.

\section{Biomechanical parameters measured by the measuring system}

The test stand component of the investigated human - machine system makes it possible to measure a number of parameters which directly or indirectly describe the dynamics and kinematics of wheelchair propulsion. One of such parameters is measured by the strain gauges (Fig. 7), which support the weighing pan. The sensors are performing continuous measurements with a specified frequency. Therefore, it is possible to measure changes in loads applied to strain gauges while performing activities resulting from normal wheelchair operation, for instance as a result of propelling it.

By using appropriate mathematical transformations we can determine changes in the position of the centre of gravity on horizontal plane XY (Fig. 8). Based on the measurement data ellipses are delimited in which the centre of gravity of the studied system may be positioned depending on the activity performed while driving the wheelchair. The results obtained in this way are used to verify the stability of the system and to determine the loads applied to wheelchair wheels. The designed test stand also allows to measure kinematic 
parameters such as speed and acceleration. The output value from the test stand is a speed measure in time (Fig. 9A). It serves as a basis for determining the time intervals for drive and return phases (Fig. 10). These intervals are plotted on the wheelchair speed function graph.

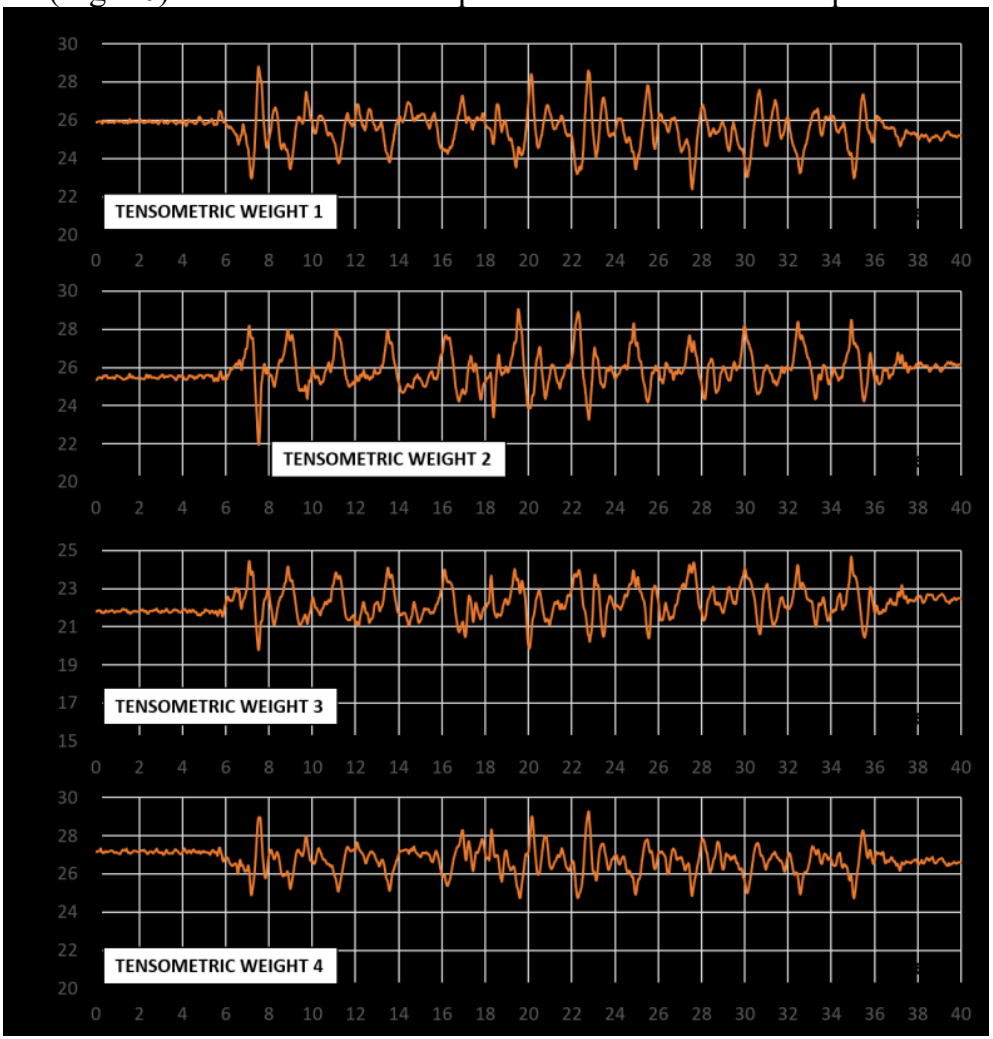

Fig. 7. Distribution of loads applied by the wheelchair with operator to individual strain gauges of the test stand

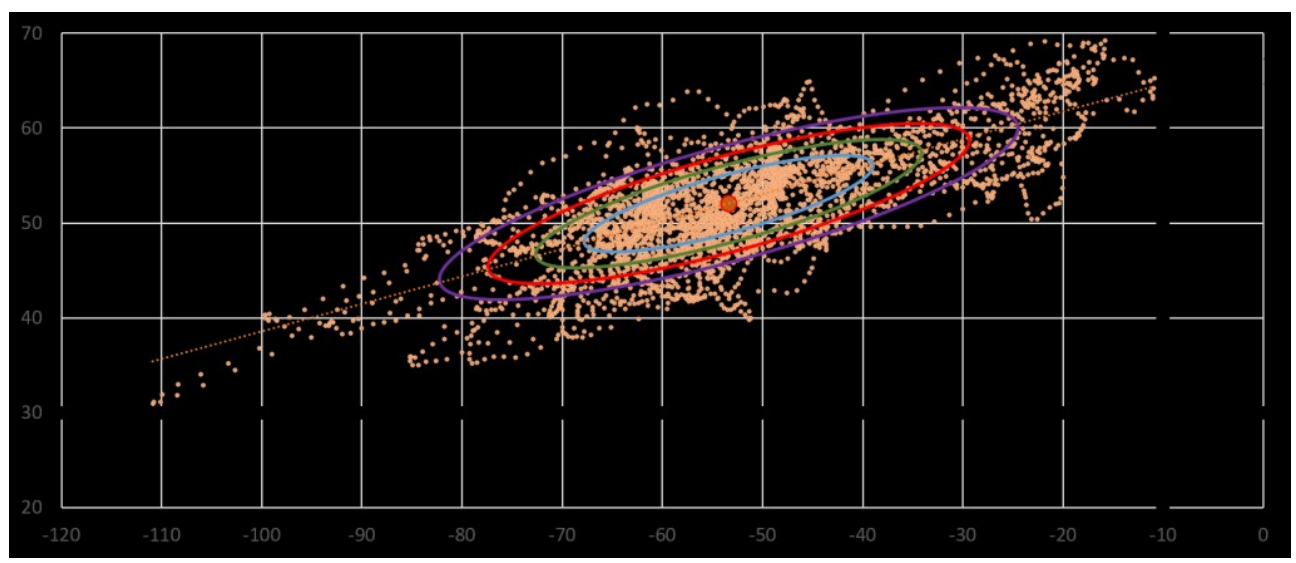

Fig. 8. Graph of the changes in the position of the centre of gravity of the man-wheelchair system

They are used to split the speed graph into parts. In the drive phase data measured at the test stand using encoders are utilized. While in the return phase the speed of the wheelchair is determined analytically. Occurrence of fixed deceleration was assumed for this phase. The value of acceleration of deceleration was determined at a separate test stand module. 
Transformations of the speed function result in obtaining the actual wheelchair speed function curves (Figs. $9 \mathrm{~B}$ and C).

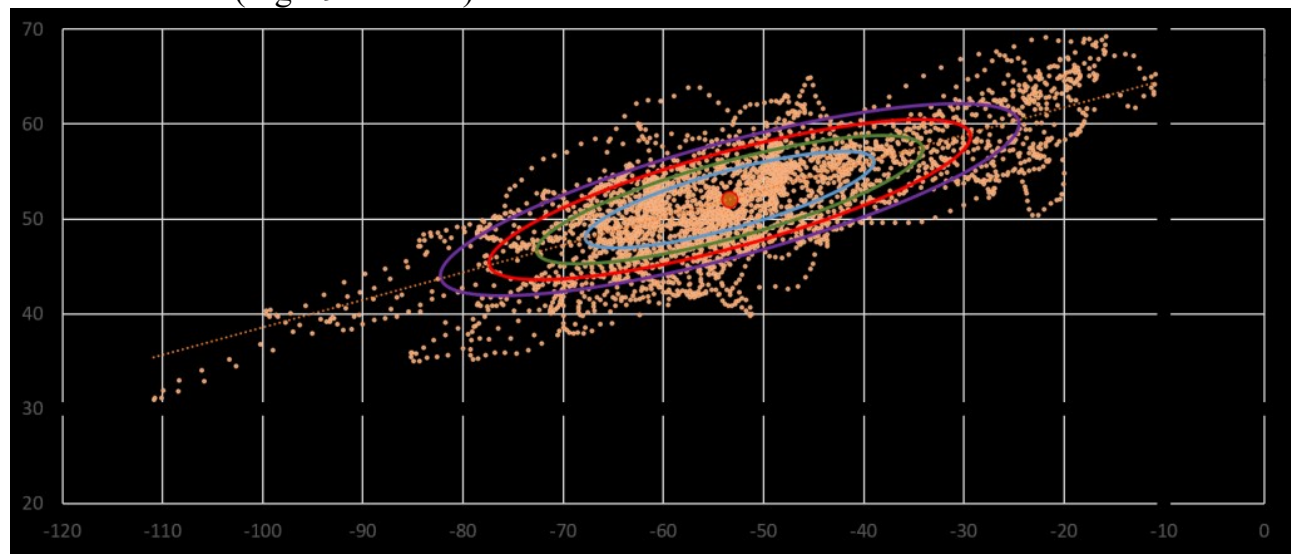

Fig. 9. Graphs of wheelchair speed measured at the test stand: A - data measured at test stand, B - data with determined speed values in return phase, $\mathrm{C}$ - smoothed wheelchair speed curve

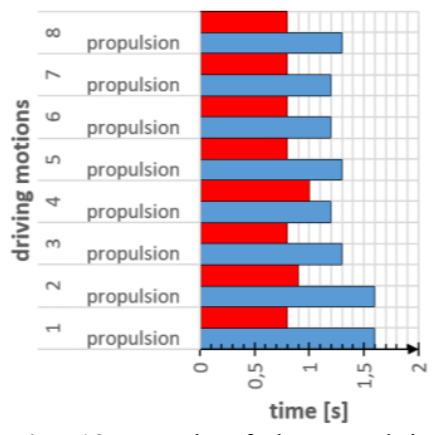

Fig. 10. Graph of the speed in time intervals of the drive and return phases in subsequent propulsion motion cycles

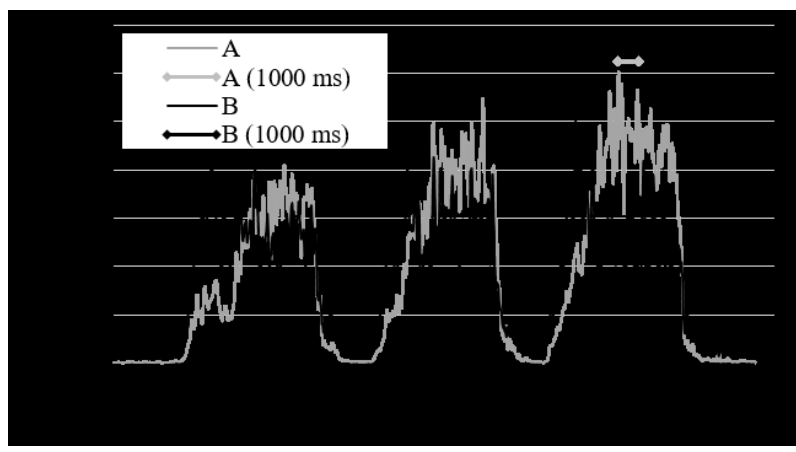

Fig. 11. An example of EMG results

Electromyographic muscle activity is another biomechanical parameter measured using the test stand (Fig. 11). The analysis of this parameter describes the use of the muscular system in the process of wheelchair propulsion. It allows to compare different types of wheelchairs in terms of muscular system use. As regards the EMG measurement the test stand acts as a system generating excitations and receiving the revolutions from the wheelchair drive wheels over the duration of the test.

\section{Summing up and conclusions}

Designing of a test stand for the investigation of wheelchair propulsion biomechanics boils down to solving two fundamental problems: the problem of wheelchair motion simulation and the problem of biomechanical parameters measurement. In the test stand described in this paper wheelchair motion is simulated with the use of a system of driving rollers, equipped with motors generating kinematic excitations and a system tilting the wheelchair frame from the horizontal position. The biomechanical parameters are measured by encoders counting wheelchair wheel revolutions, by strain gauges measuring distribution of loads generated by 
the weight of the wheelchair and by an surface EMG machine for the measurement of electromyographic activity of muscles.

The key element of the research is processing of the data collected during the tests. The processing involves for instance determining the centre of gravity of the human body based on the results of the measurements taken by strain gauges, or projection of wheelchair speed in the return phase of the propulsion cycle. In the latter case, due to the test stand's design and internal resistances present, in the return phase, the wheelchair wheel stopped shortly after the completion of the drive phase. Therefore it was necessary to analytically determine the wheelchair speed during the return phase and to simulate it in appropriate time intervals of the entire propulsion cycle.

The designed test stand makes it possible to measure biomechanical parameters such as loads applied to wheelchair wheels, position of the centre of gravity, wheelchair speed, acceleration, propelling force, electromyographic activity of the muscles and other analytically determinable parameters. This data range allows for a comprehensive comparison of different types of wheelchairs and forms a basis for the modification and adjustment of the existing wheelchair designs with the objective of improving their functionality.

\section{References}

1. V.L. Goosey-Tolfrey, A.D. Moss, Wheelchair velocity of tennis players during propulsion with and without the use of racquets. Adapted physical activity quarterly, Vol. 22, 291-301 (2005)

2. S.D. Shimada, R.N. Robertson, M.L. Bonninger, R.A. Cooper, Kinematic characterization of wheelchair propulsion. Journal of Rehabilitation Research and Development, Vol. 35 No. 2, 210-218 (1998)

3. Y. Vanlandewijck, D. Theisen, D. Daly, Wheelchair Propulsion Biomechanics. Implications for Wheelchair Sports. Sports Med Vol. 31 No. 5, 339-367 (2001)

4. A. de Barros Lombardi Jr., F.G. Dedini, Biomechanical model for the determination of forces on upper-extremity members during standard wheelchair propulsion. Mathematical and Computer Modelling, Vol. 49, 1288-1294 (2009)

5. A.M. Kwarciak, M. Yarossi, A. Ramanujam, T.A. Dyson-Hudson, S.A. Sisto, Evaluation of wheelchair tire rolling resistance using dynamometer-based coast-down tests. Journal of Rehabilitation Research and Development, Vol. 46, No. 7, 931-938 (2009)

6. B. Wieczorek, J. Górecki, M. Kukla, D. Wojtkowiak, D. Wilczyński, Urządzenie do symulacji warunków eksploatacji $i$ pomiaru parametrów dynamicznych wózka inwalidzkiego. Patent claim P.424482 filed to the Patent Office of the Republic of Poland (2018) 